\title{
Single-docking robotic assisted proctectomy for rectal cancer below peritoneal reflection: a propensity score matching analysis
}

\author{
Tao Zhang", Zijia Song", Yaqi Zhang", Feng Ye, Xi Cheng, Shaodong Wang, Xiaoqian Jing, Xiaopin Ji, \\ Ren Zhao \\ Department of General Surgery, Ruijin Hospital, Shanghai Jiao Tong University School of Medicine, Shanghai, China \\ Contributions: (I) Conception and design: T Zhang, Z Song; (II) Administrative support: T Zhang, Y Zhang, X Cheng; (III) Provision of study \\ materials or patients: R Zhao, X Ji; (IV) Collection and assembly of data: X Jing, T Zhang, S Wang; (V) Data analysis and interpretation: Z Song, Y \\ Zhang; (VI) Manuscript writing: All authors; (VII) Final approval of manuscript: All authors. \\ "These authors contributed equally to this work. \\ Correspondence to: Ren Zhao, MD, PHD, FACS; Xiaopin Ji, MD, PhD; Xiaoqian Jing, MD, PhD. Department of General Surgery, Ruijin Hospital, Shanghai \\ Jiao Tong University School of Medicine, Shanghai 200025, China. Email: zhaorensurgeon@aliyun.com; jxp11156@rjh.com; 742842063@qq.com.
}

Background: The aim of this study was to compare the short and long-term outcomes of robotic assisted proctectomy (RP) and laparoscopic assisted proctectomy (LP) for rectal cancer below the peritoneal reflection using propensity score matching (PSM) analysis.

Methods: We evaluated the medical records of 200 patients who underwent proctectomy for rectal cancer below the peritoneal reflection through a robotic $(n=81)$ or laparoscopic $(n=119)$ approach between Jan 2015 and Dec 2017. The data were prospectively collected, and the patients were matched at a ratio of 1:1 according to age, sex, body mass index (BMI), previous abdominal surgeries, comorbidities, American Society of Anesthesiologist score $(\leq 2 />2)$, and pathologic stage.

Results: After matching, each group included 74 patients. Compared to the LP group, the RP group showed shorter postoperative hospital stays (PHS) [7 $( \pm 2)$ vs. $9( \pm 2.3) \mathrm{d}, \mathrm{P}=0.003]$, shorter time to liquid $\operatorname{diet}[3( \pm 2)$ vs. $5( \pm 3) \mathrm{d}, \mathrm{P}<0.001]$, and shorter time to removal of catheter $[6( \pm 2) v s .7( \pm 2.3) \mathrm{d}, \mathrm{p}=0.014]$. The operative expense was higher in the RP group $[8,365( \pm 1,600)$ vs. $6,922( \pm 1,220) \mathrm{RMB}, \mathrm{P}<0.001]$. The operation time, estimated blood loss, postoperative complications, and pathologic outcomes were similar between the two groups. No conversion to laparotomy, readmission, or mortality was observed in either group within 30 days after surgery. The 3-year disease-free survival (DFS) were $75.2 \%$ and $88.3 \%(\mathrm{P}=0.070)$, and overall survival (OS) were $92.9 \%$ and $93.7 \%(\mathrm{P}=0.810)$ in the RP and the LP groups, respectively and the risk of low anterior resection syndrome (LARS) was lower in the RP group (OR =0.304, 95\% CI: 0.124$0.745, \mathrm{P}=0.009)$.

Conclusions: Compared to LP, RP is worth recommending as it has long-term survival, faster postoperative recovery, and a lower risk of LARS in patients with rectal cancer below the peritoneal reflection.

Keywords: Rectal cancer; robotic assisted proctectomy (RP); laparoscopic assisted proctectomy (LP); short-term outcomes; long-term outcomes

Submitted Apr 30, 2021. Accepted for publication Jun 16, 2021.

doi: $10.21037 / \mathrm{atm}-21-2744$

View this article at: https://dx.doi.org/10.21037/atm-21-2744 


\section{Introduction}

Colorectal cancer (CRC) is the third most common malignancy in the world. The proportion of new cancer cases and cancer death of CRC was $10 \%$ and $9.4 \%$ according to Global Cancer Statistics 2020 (1). Among the different locations, mid-low rectal cancer is known as requiring a relatively high level of surgical expertise, as both the resection margin (distal margin and circumferential margin) and lymph node dissection in a relatively narrow space.

Through almost two decades of development, robotic surgery has been increasingly applied in rectal surgery owing to its stability and dexterity, 3-dimensional highdefinition display, and precision and accuracy in anatomical dissection. However, robotic surgery is expensive and private insurers may not fully reimburse its cost. Despite this, robotic surgery for rectal cancer has continued to gain global utilization, and some clinical analyses report robotic rectal resection is a safe and adequate technique for the treatment of rectal cancer. Further, it was strongly associated with better short-term outcomes over laparoscopic surgery, and even allowed for the preservation of urinary and sexual functions in patients with mid-low rectal cancer $(2,3)$.

In the present study, we used a propensity score match (PSM) method with real world data from a single center, those who with $\mathrm{T}$ stage under $\mathrm{T} 3$ and received no neoadjuvant radiotherapy were included, with the aim of retrospectively comparing the short-term and longterm outcomes of robotic assisted proctectomy (RP) and laparoscopic assisted proctectomy (LP) for rectal cancer below the peritoneal reflection.

We present the following article in accordance with the STROBE reporting checklist (available at https://dx.doi. org/10.21037/atm-21-2744).

\section{Methods}

\section{Patients and data collection}

The study was conducted in accordance with the Declaration of Helsinki (as revised in 2013). It was approved by institutional ethics board of Ruijin Hospital, Shanghai Jiao Tong University School of Medicine. (No.: 109/2017) and informed consent was taken from all individual participants. Following institutional review board approval, a retrospective chart review was performed on consecutive patients who underwent RP and LP for rectal adenocarcinoma from Jan 2015 to Dec 2017 at the Department of General Surgery, Ruijin Hospital, Shanghai
Jiao Tong University School of Medicine in Shanghai, China. The exclusion criteria were abdominal perineal resection, the use of new adjuvant chemoradiotherapy, stage IV rectal cancer, T4 tumors, tumors above the peritoneal reflection, the concomitant presence of other malignant tumors, and incomplete records for review. After screening, 200 patients (RP:81; LP:119) were included in the study.

Clinicopathologic information and perioperative outcomes were obtained from medical records, including sex, age, body mass index (BMI), ASA grade, comorbidities, previous abdominal surgery, operation time, estimated blood loss, time to liquid diet, length of postoperative hospital stay (PHS), conversion to laparotomy, temporary terminal ileostomy, postoperative complications, perioperative mortality, tumor size, histology, lymph nodes harvest, proximal and distal resection margins, circumferential resection margin (CRM), metastatic lymph node, neurovascular invasion, anastomotic height, and pathologic stage according to the 8th Edition of the AJCC Cancer Staging Manual. Postoperative complications were graded according to the Clavien-Dindo classification.

Follow-up surveillance was consistent with the National Comprehensive Cancer Network (NCCN) guidelines, and recurrence was confirmed by radiological or histological methods. Patients with Stage III or Stage II lesions with high risk were routinely sent to chemotherapy or radiotherapy for further treatment according to the guidelines. A low anterior resection syndrome (LARS) questionnaire was collected 18 months after surgery.

\section{Surgical procedure}

All operations were carried out by the same wellexperienced and qualified surgical team, who performed more than $400 \mathrm{RP}$ or LP surgeries per year. Bowel preparation was the same between the two groups including 1-2 days of liquid diet and polyethylene glycol electrolyte solution, and prophylactic antibiotics the day before operation.

In the RP group, a single-docking technique with five ports to fulfill the whole process was performed (Figure 1). As the tumor was located below the peritoneal reflection, the anastomosis could be quite low, and high ligation for lymph node dissection was required, which resulted in the left colic artery not being preserved in most patients. The splenic flexure of the colon was not routinely mobilized, depending on the tension of the anastomosis. Following the principle of total mesorectal excision (TME), 


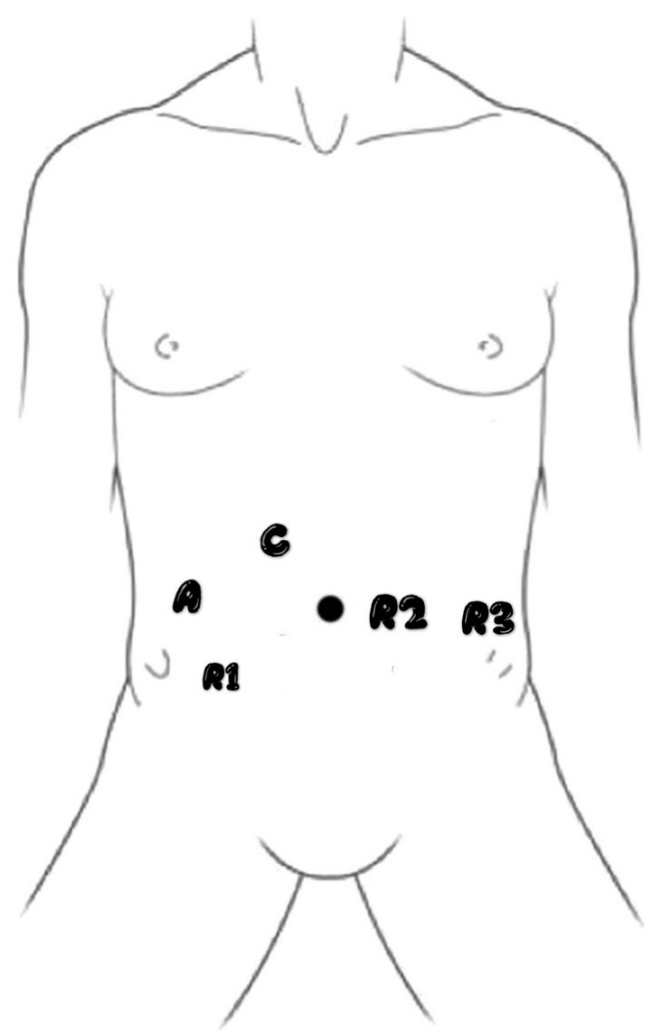

Figure 1 Port sites in the RP group. A, assistant; R1, 2, 3, robotic 1, 2, 3; C, camera. RP, robotic assisted proctectomy.

anterior resection (AR) with a double-staple technique was performed accordingly. The tumor specimen was extracted through a small hypogastric midline incision with protection of the incision (Figure 2).

A temporary terminal ileostomy was not routinely performed except in cases in which the surgeon considered the patient had a substantial risk of anastomotic leakage. The dissected pelvic peritoneum was not sutured, and one or two drainage cannulas were placed near the anastomosis for detecting leakage. Once leakage occurred, the cannula was flushed, and the faeces drained to prevent local infection.

As the laparoscopic procedure was similar to the robotic procedure and is well documented elsewhere, it is not described in this paper. All patients were supervised by the same treatment team according to unified standards.

\section{Matching}

Propensity Score Matching (PSM) is a statistical method used to process observational study data. Due to various reasons, there are many data biases and confounding variables in observational study. The method of propensity score matching is to reduce the influence of these biases and confounding variables, so as to make more reasonable comparison between the experimental group and the control group. We applied 1:1 PSM by using bivariate logistic regression, and age, sex, BMI, ASA grade, comorbidities, previous abdominal surgery, and pathologic stage were selected as covariates.

\section{Statistical analysis}

Statistical analysis was performed with SPSS (version 23.0, SPSS Inc. Chicago, IL, USA), and statistically significant differences were evaluated using the Student's $t$-test, MannWhitney $\mathrm{U}$ test, $\chi^{2}$ test, and Fisher exact test, as appropriate. Disease-free survival (DFS) rates and overall survival (OS) were estimated by the Kaplan-Meier method and compared by the log-rank test. LARS was predicted by binary logistic regression analyses and a $\mathrm{P}$ value $<0.05$ was considered statistically significant.

\section{Results}

\section{Baseline characteristics}

Prior to matching, patients in the two groups showed great significance in comorbidity [20 (24.7\%) vs. 50 (42\%) $\mathrm{P}=0.015]$ and previous abdominal surgery $[4$ (4.9\%) vs. $18(15.1 \%) \mathrm{P}=0.036]$. However, after matching, each group comprised of 74 patients, and differences in patient characteristic were eliminated (Table 1).

\section{Intraoperative and perioperative outcomes}

Robotic surgery was superior to laparoscopic surgery in time to liquid diet $[3( \pm 2)$ vs. $5( \pm 3), \mathrm{P}<0.001]$, removal of catheters $[6( \pm 2)$ vs. $7( \pm 2.3), \mathrm{P}=0.014]$, and the length of PHS [7 $( \pm 2)$ vs. $9( \pm 2.3), \mathrm{P}=0.003]$ (Table 2). There were no significant differences between the RP and LP groups in operation time, estimated blood loss, temporary terminal ileostomy, anastomotic height, and postoperative complications. No cases were converted to laparotomy, and there were neither readmissions nor mortality within 30 days after surgery in either group. The operation time was measured from the beginning of anesthesia until the end of all surgical procedures, and since draping and docking commenced when the anesthesiologist started infusion, this added no extra time. Most complications 


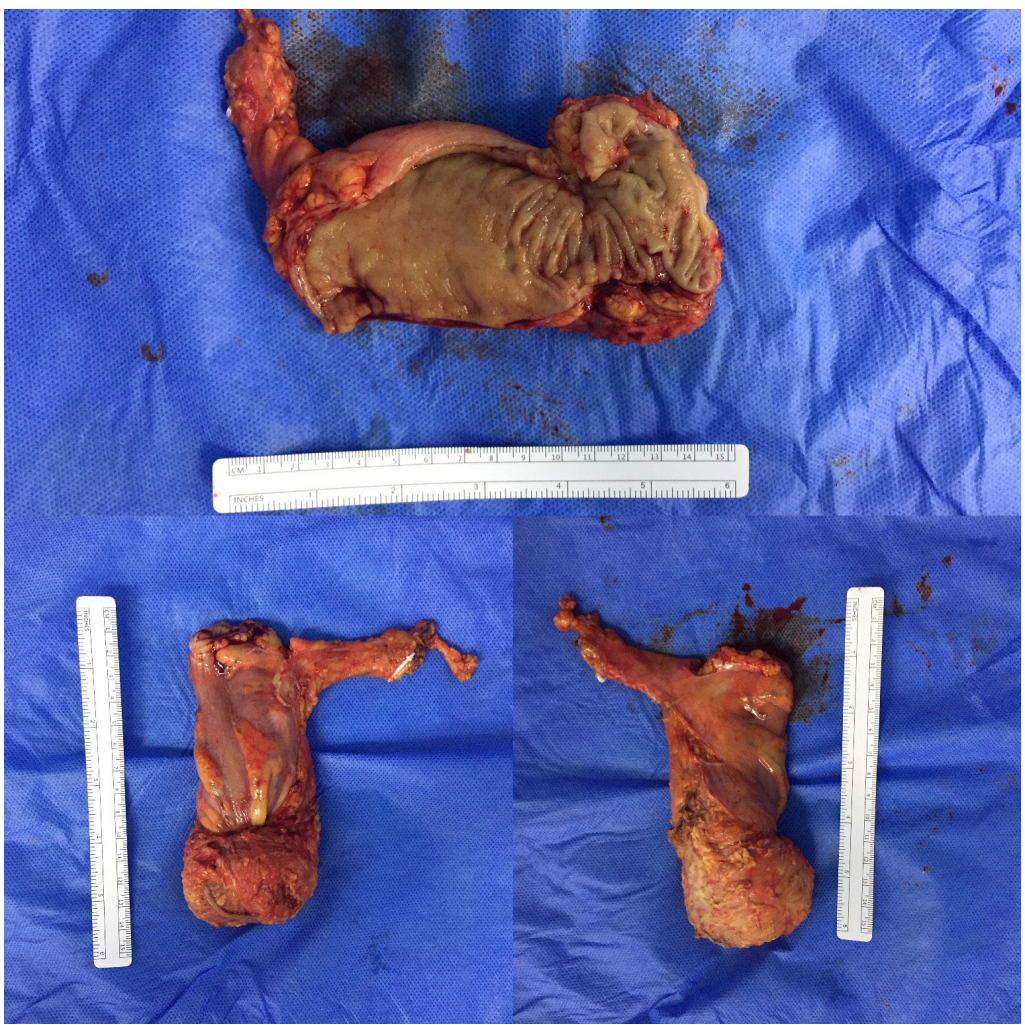

Figure 2 A typical total mesorectal excision specimen.

Table 1 Baseline characteristics

\begin{tabular}{|c|c|c|c|c|c|c|}
\hline Characteristics & \multicolumn{3}{|c|}{ Overall } & \multicolumn{3}{|c|}{ After matching } \\
\hline Age, median [IQR], years & 64 [15] & 63 [16] & 0.364 & 64 [14] & 61.5 [13] & 0.594 \\
\hline Sex, n (\%) & & & 1 & & & 1 \\
\hline Male & $53(65.4)$ & $77(64.7)$ & & $48(64.9)$ & $48(64.9)$ & \\
\hline BMI, median [IQR], kg/m² & $23.30[4.53]$ & 23.84 [4.22] & 0.511 & $23.17[4.18]$ & 23.28 [3.59] & 0.798 \\
\hline ASA grade, $n(\%)$ & & & 0.128 & & & 1 \\
\hline$\leq 2$ & 79 (97.5) & $109(91.6)$ & & $72(97.3)$ & 72 (97.3) & \\
\hline$>2$ & $2(2.5)$ & $10(8.4)$ & & $2(2.7)$ & $2(2.7)$ & \\
\hline Pathologic stage, n (\%) & & & 0.232 & & & 0.868 \\
\hline 0,1 & $15(18.5)$ & $31(26.0)$ & & $14(18.9)$ & $17(23.0)$ & \\
\hline II & $34(42.0)$ & 37 (31.1) & & $28(37.8)$ & $26(35.1)$ & \\
\hline III & 32 (39.5) & $51(42.9)$ & & $32(43.2)$ & $31(41.9)$ & \\
\hline
\end{tabular}

IQR, interquartile range; BMI, body mass index; ASA, The American Society of Anesthesiologists. 
Table 2 Intraoperative and perioperative outcomes

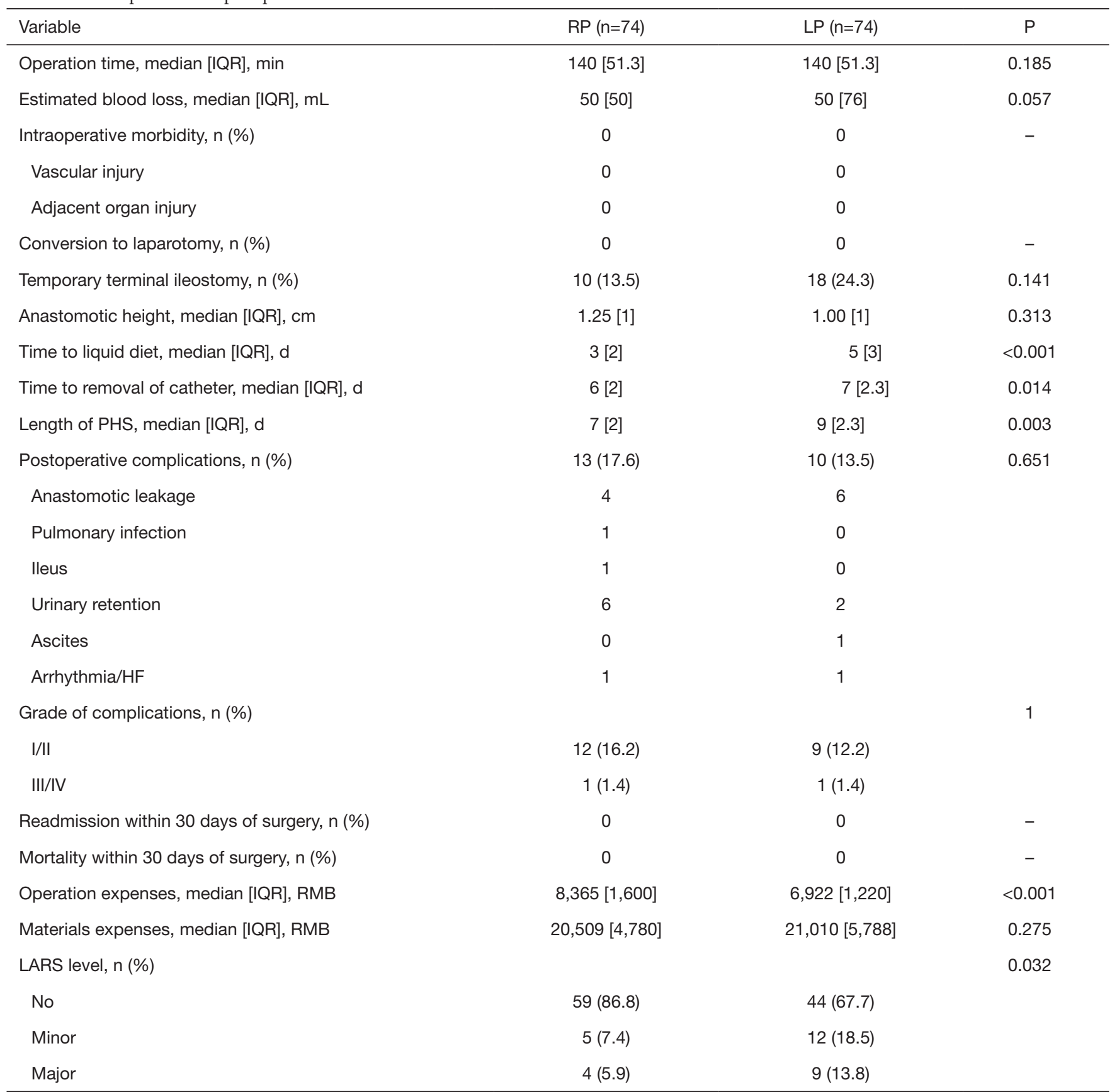

PHS, postoperative hospital stay; HF, heart failure; IQR, interquartile range; LARS, low anterior resection syndrome; RP, robotic assisted proctectomy; LP, laparoscopic assisted proctectomy.

were grade I/II and included urinary retention with a delay of Foley catheter removal in less than a week, pulmonary infection, ileus, and arrythmias, while ascites occurred in one patient pre-comorbid with cirrhosis. There were four cases $(5.4 \%)$ of anastomotic leakage in the RP group and six $(8.1 \%)$ in the LP group, while one patient $(1.4 \%)$ in the LP group required an extra diversion stoma to control the leakage and was considered a grade IIIb case. Other cases were cured by flush and drainage through the catheter. One aged patient (1.4\%) in the RP group with poor preoperative 
Table 3 Pathologic and oncologic outcomes

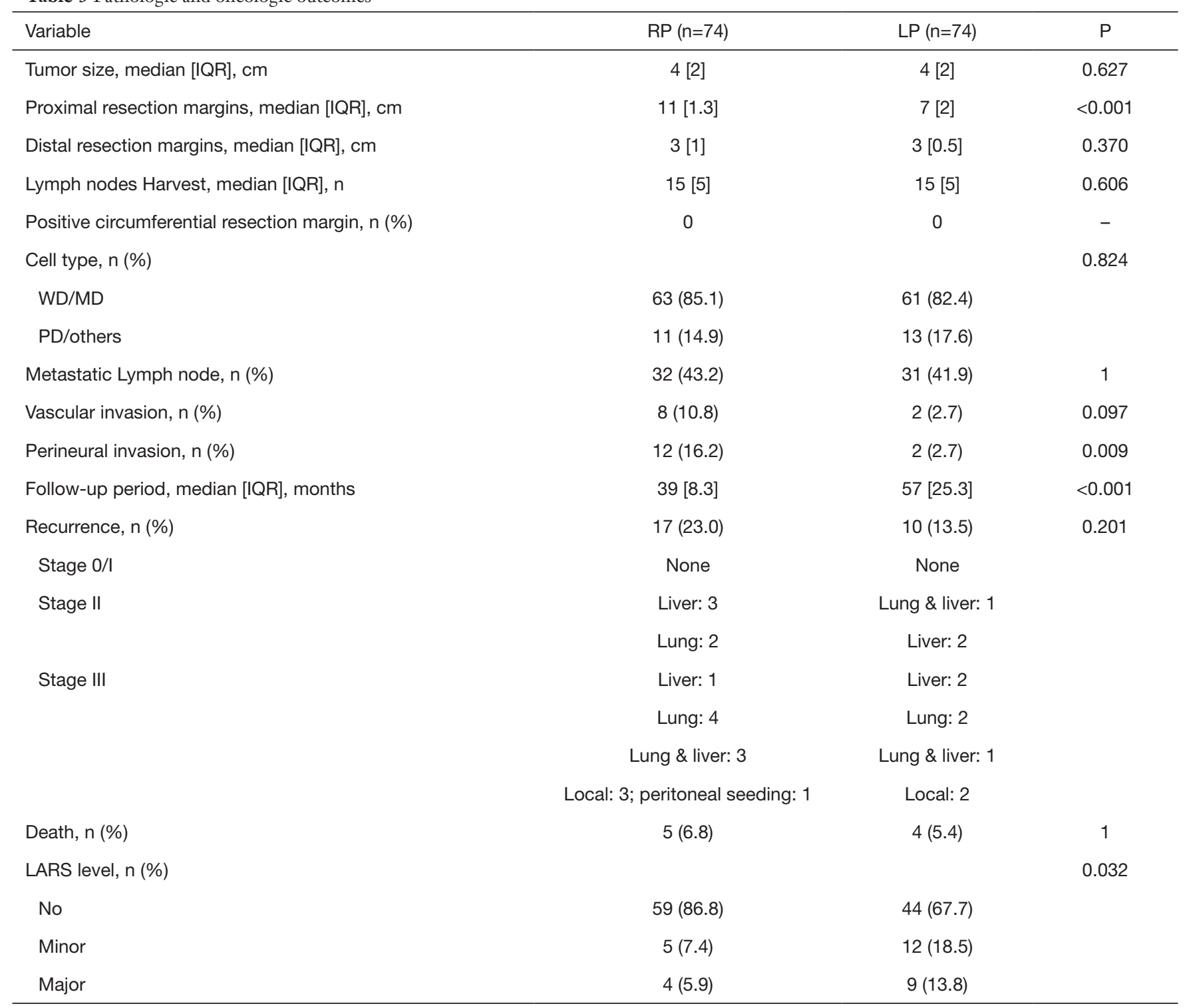

IQR, interquartile range; WD, well differentiated; MD, moderately differentiated; PD, poorly differentiated; LARS, low anterior resection syndrome; RP, robotic assisted proctectomy; LP, laparoscopic assisted proctectomy.

heart function suffered heart failure after surgery and was sent to Intensive Care Unit to rehabilitate. While the operative material expense, including disposable instruments and staplers, was not different between the groups, the operative ongoing maintenance was higher in the RP group with statistical significance $[8,365( \pm 1,600) v s .6,922$ $( \pm 1,220) \mathrm{RMB}, \mathrm{P}<0.001]$. The extra expense associated with the machine running fee in robotic surgery amounted to about 30,000 RMB (4,200 US dollars), although private insurance covered this cost for some patients.

\section{Pathologic and long-term outcomes}

The tumor size, CRM, distal resection margins, lymph nodes harvest, histology, metastatic lymph node, and vascular invasion did not differ significantly between the two groups (Table 3). A longer proximal resection margin $[11( \pm 1.3)$ vs. 7 $( \pm 2) \mathrm{cm}, \mathrm{P}<0.001]$ and more perineural invasion $[12( \pm 16.2)$ vs. $2( \pm 2.7), \mathrm{P}=0.009$ ] were found in the $\mathrm{RP}$ group. The median follow-up period was 39 months in the RP group and 57 months in the LP group $(\mathrm{P}<0.001)$, the 3 -year DFS 


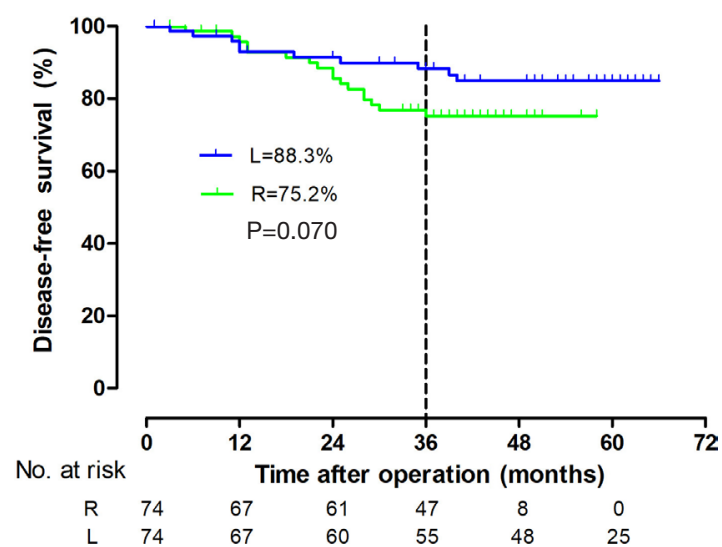

Figure 3 Kaplan-Meier analysis of disease-free survival.

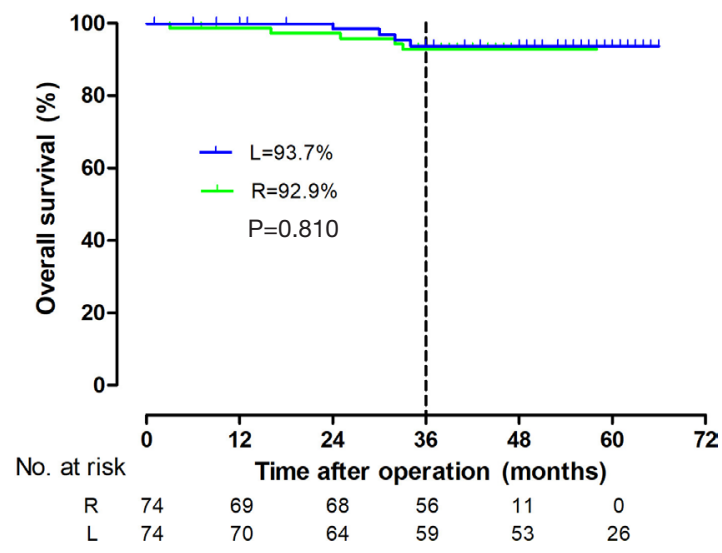

Figure 4 Kaplan-Meier analysis of overall survival.

were $75.2 \%$ and $88.3 \%(\mathrm{P}=0.070$, Figure 3$)$, and the OS were $92.9 \%$ and $93.7 \%(\mathrm{P}=0.810$, Figure 4$)$ in the $\mathrm{RP}$ and the $\mathrm{LP}$ groups, respectively. The total LARS incidence (assessed 18 months after surgery) was higher in the LP group [RP: minor 5 (7.1\%), major 4 (5.7\%); LP: minor 12 (17.9\%), major $9(13.4 \%), \mathrm{P}=0.032]$, evaluated by LARS score. After univariate analysis was performed (Table 4), surgical approach (RP or LP), T stage (Tis/T1/T2 or T3), and age were selected as covariates in the binary logistic regression to evaluate the predictors of LARS. The results (Table 5) showed that RP patients had a lower risk of LARS than LP patients (OR $=0.304,95 \%$ CI: $0.124-0.745, \mathrm{P}=0.009$ ).

\section{Discussion}

Although the question of whether laparoscopic surgery is equivalent to open procedures in the treatment of mid or low proctectomy remains controversial, several well-known multicentral randomized trials had proven the noninferiority of laparoscopic proctectomy compared to open procedures in recent years (4-8). In 2009, the UK Medical Research Council and National Institute of Health Research, through the Efficacy and Mechanism Evaluation Program, funded the Robotic vs. Laparoscopic Resection for Rectal Cancer (ROLARR) trial to undertake an evaluation of the safety, efficacy, and short- and long-term outcomes of roboticassisted $v s$. conventional laparoscopic rectal cancer surgery (9). No difference between the treatment groups was observed for longer-term outcomes, disease-free, and OS, and the short-term outcomes were complicated. This demonstrated that among patients with rectal adenocarcinoma suitable for curative resection, robotic-assisted laparoscopic surgery, as compared with conventional laparoscopic surgery, did not significantly reduce the risk of conversion to open laparotomy. These findings suggest that when performed by surgeons with varying experience, robotic-assisted laparoscopic surgery does not confer an advantage in rectal cancer resection $(3,10,11)$.

In our study, we focused on surgical procedures for rectal cancer below the peritoneal reflection, which guaranteed it to be a study of true mid-low rectal surgery. We found robotic assisted surgery manifested an overall faster recovery when compared to laparoscopic procedures, in the time of catheter removal, time to liquid diet, and length of PHS. Robotic assisted surgery did not increase the incidence of conversion to laparotomy, postoperative complications, operation time, intraoperative blood loss, readmission, or mortality within 30 days of surgery.

RP could also reach the standard of TME and showed no difference to laparoscopic procedures. Other studies have reported that the CRM had an overall positive rate of around $5 \%$ (9), with no difference in robotic and laparoscopic groups. However, in the present study, there were no positive CRM patients in either group, which may be due to the fine-selection of patients who received robotic surgery (T1-T3) and the PSM statistical process. The OS were quite promising in demonstrating equivalent longterm outcomes of the surgical methods. However, in the 3 -year DFS, although there's no statistic difference between 2 groups, the curve seemed separated after 24 months. Looking into the data, we found a higher occurrence of perineural invasion, this may cause more lung metastasis in robotic group.

A robotic surgical system provides outstanding dexterity, stability, and accuracy. Accordingly, it is particularly useful 
Table 4 Univariate analysis of LARS after AR in 137 patients

\begin{tabular}{|c|c|c|c|c|c|}
\hline Factor & Total & No/minor LARS & Major LARS & Statistics & $P$ value \\
\hline Robotic & 70 & 61 & 9 & & \\
\hline Laparoscopic & 67 & 46 & 21 & $\chi^{2}=6.840$ & 0.009 \\
\hline \multicolumn{6}{|l|}{ Sex } \\
\hline Age & 137 & 60.79 & 63.57 & $\mathrm{~T}=-1.188$ & 0.237 \\
\hline BMI & 137 & 23.17 & 23.54 & $\mathrm{~T}=-0.611$ & 0.542 \\
\hline \multicolumn{6}{|l|}{ Surgical history } \\
\hline 1 & 30 & 22 & 8 & & \\
\hline II & 53 & 41 & 12 & & \\
\hline III & 54 & 44 & 10 & $\chi^{2}=0.777$ & 0.678 \\
\hline \multicolumn{6}{|l|}{ T stage } \\
\hline 1 & 14 & 7 & 7 & & \\
\hline 2 & 20 & 16 & 4 & & \\
\hline 3 & 103 & 83 & 19 & $\chi^{2}=7.224$ & 0.027 \\
\hline \multicolumn{6}{|l|}{ Vascular invasion } \\
\hline Blood loss (mL) & 137 & 73.87 & 54.00 & $\mathrm{~T}=1.265$ & 0.208 \\
\hline Operation time (min) & 137 & 148.12 & 148.67 & $\mathrm{~T}=-0.059$ & 0.953 \\
\hline Anastomotic height (cm) & 137 & 1.360 & 1.317 & $\mathrm{~T}=0.338$ & 0.736 \\
\hline
\end{tabular}

LARS, low anterior resection syndrome; AR, anterior resection.

Table 5 Multivariate analysis of LARS after AR in 137 patients

\begin{tabular}{lccc}
\hline Factor & OR & $95 \% \mathrm{Cl}$ & $\mathrm{P}$ value \\
\hline Surgical approach & 0.311 & $0.125-0.772$ & 0.012 \\
T stage & - & - & 0.073 \\
1 & Ref & - & - \\
2 & 0.255 & $0.053-1.221$ & 0.087 \\
3 & 0.252 & $0.076-0.834$ & 0.024 \\
Age & 1.030 & $0.991-1.072$ & 0.137 \\
\hline
\end{tabular}

LARS, low anterior resection syndrome; AR, anterior resection. in dissecting tissue in a narrow space such as the pelvis, and in the protection of important nerves and vessels. It is reported that scores of the European Organization for Research and Treatment of Cancer Quality of Life (EORTC QLQ C30) and EORTC QLQ CR38 were similar in both robotic and laparoscopic groups, but in the EORTC QLQ CR38 questionnaire, sexual function 12 months postoperatively was better in the robot-assisted group than in the laparoscopic group (12). Similarly, Chang et al. (3) found urinary function and general sexual satisfaction 
decreased significantly 1 month after robotic surgery for both sexes. However, both parameters subsequently increased progressively, and the values 1 year after surgery were comparable to those measured before. Some studies (13-19), found a significant prevalence of LARS following oncological rectal resection, and a low anastomotic height or history of radiotherapy were major risk factors. Patients with LARS often experience either a pattern of urgency and incontinence, or alternately, obstructed defecation. Bowel adaptation is thought to occur by about 18 months post operatively, after which, further improvement with time is unlikely, and there is no effective medical treatment. In reviewing the charts of patients in our cohort, we found the incidence of LARS was much higher in laparoscopic groups, and the univariant analysis and multivariant analysis confirmed that the risk of LARS in robotic assisted surgery was lower than that in laparoscopic assisted surgery. Combined with the above studies, we found that RP had better long-term postoperative functional recovery for patients with rectal cancer below peritoneal reflection and is worthy of recommendation.

The only limitation of robotic rectal surgery could be the relatively high expense. Some patients could not accept the extra expense due to their economic situation. However, a new policy is announced this year in some city of China, i.e., almost $80 \%$ of the surgical expense of robotic rectal surgery can be reimbursed by the national health insurance. So, in the near future, more patients would choose robotic surgery.

This study had several limitations, including its small sample size and that it was conducted at a single center. In addition, patients with stage IV rectal cancer, T4 tumor, and those receiving new adjuvant chemoradiotherapy were not included, which could possibly influence the DFS and OS results and although the follow-up period of both groups exceeded 36 months, the median followup period of robotic surgery was shorter than laparoscopic surgery. Finally, as this is a retrospective study, the potential selection bias and confounding bias could not be completely excluded despite the use of PSM.

\section{Conclusions}

$\mathrm{RP}$ below the peritoneal reflection has the advantage of fast recovery compared to laparoscopic assisted surgery. The risk of LARS in robotic assisted surgery was lower than that in laparoscopic assisted surgery and the long-term survival of both was equivalent. The results of this study suggest RP is worthy of recommendation in patients with rectal cancer below the peritoneal reflection.

\section{Acknowledgments}

Funding: The study was supported by the National Natural Science Foundation of China [81902374 (XJ)], [82002475 (XC)], the Shanghai Sailing Program [20YF1427700 (XC)], the Shanghai Hospital Development Center [16CR2064B (RZ)], the Shanghai Municipal Health Construction Commission [201540026 (RZ)], the Youth Development Program of Ruijin Hospital, the Shanghai Jiaotong University School of Medicine [2019QNPY01010 (TZ)].

\section{Footnote}

Reporting Checklist: The authors have completed the STROBE reporting checklist. Available at https://dx.doi. org/10.21037/atm-21-2744

Data Sharing Statement: Available at https://dx.doi. org/10.21037/atm-21-2744

Conflicts of Interest: All authors have completed the ICMJE uniform disclosure form (available at https://dx.doi. org/10.21037/atm-21-2744). The authors have no conflicts of interest to declare.

Ethical Statement: The authors are accountable for all aspects of the work in ensuring that questions related to the accuracy or integrity of any part of the work are appropriately investigated and resolved. The study was conducted in accordance with the Declaration of Helsinki 2013. The study was approved by institutional ethics board of Ruijin Hospital, Shanghai Jiao Tong University School of Medicine (No.: 109/2017) and informed consent was taken from all individual participants.

Open Access Statement: This is an Open Access article distributed in accordance with the Creative Commons Attribution-NonCommercial-NoDerivs 4.0 International License (CC BY-NC-ND 4.0), which permits the noncommercial replication and distribution of the article with the strict proviso that no changes or edits are made and the original work is properly cited (including links to both the formal publication through the relevant DOI and the license). See: https://creativecommons.org/ licenses/by-nc-nd/4.0/. 


\section{References}

1. Sung H, Ferlay J, Siegel RL, et al. Global Cancer Statistics 2020: GLOBOCAN Estimates of Incidence and Mortality Worldwide for 36 Cancers in 185 Countries. CA Cancer J Clin 2021;71:209-49.

2. Sammour T, Malakorn S, Bednarski BK, et al. Oncological Outcomes After Robotic Proctectomy for Rectal Cancer: Analysis of a Prospective Database. Ann Surg 2018;267:521-6.

3. Chang W, Wei Y, Ren L, et al. Short-term and long-term outcomes of robotic rectal surgery-from the real word data of 1145 consecutive cases in China. Surg Endosc 2020;34:4079-88.

4. Denost Q, Adam JP, Rullier A, et al. Perineal transanal approach: a new standard for laparoscopic sphincter-saving resection in low rectal cancer, a randomized trial. Ann Surg 2014;260:993-9.

5. Hsieh C, Cologne KG. Laparoscopic Approach to Rectal Cancer-The New Standard? Front Oncol 2020;10:1239.

6. Jeong SY, Park JW, Nam BH, et al. Open versus laparoscopic surgery for mid-rectal or low-rectal cancer after neoadjuvant chemoradiotherapy (COREAN trial): survival outcomes of an open-label, non-inferiority, randomised controlled trial. Lancet Oncol 2014;15:76774. Erratum in: Lancet Oncol 2016;17:e270.

7. Fleshman J, Branda M, Sargent DJ, et al. Effect of Laparoscopic-Assisted Resection vs Open Resection of Stage II or III Rectal Cancer on Pathologic Outcomes: The ACOSOG Z6051 Randomized Clinical Trial. JAMA 2015;314:1346-55.

8. van der Pas MH, Haglind E, Cuesta MA, et al. Laparoscopic versus open surgery for rectal cancer (COLOR II): short-term outcomes of a randomised, phase 3 trial. Lancet Oncol 2013;14:210-8.

9. Jayne D, Pigazzi A, Marshall H, et al. Robotic-assisted surgery compared with laparoscopic resection surgery for rectal cancer: the ROLARR RCT. Southampton (UK): NIHR Journals Library; 2019.

Cite this article as: Zhang T, Song Z, Zhang Y, Ye F, Cheng X, Wang S, Jing X, Ji X, Zhao R. Single-docking robotic assisted proctectomy for rectal cancer below peritoneal reflection: a propensity score matching analysis. Ann Transl Med 2021;9(12):1013. doi: 10.21037/atm-21-2744
10. Jayne D, Pigazzi A, Marshall H, et al. Effect of RoboticAssisted vs Conventional Laparoscopic Surgery on Risk of Conversion to Open Laparotomy Among Patients Undergoing Resection for Rectal Cancer: The ROLARR Randomized Clinical Trial. JAMA 2017;318:1569-80.

11. Jones K, Qassem MG, Sains P, et al. Robotic total mesorectal excision for rectal cancer: A systematic review following the publication of the ROLARR trial. World J Gastrointest Oncol 2018;10:449-64.

12. Kim MJ, Park SC, Park JW, et al. Robot-assisted Versus Laparoscopic Surgery for Rectal Cancer: A Phase II Open Label Prospective Randomized Controlled Trial. Ann Surg 2018;267:243-51.

13. Croese AD, Lonie JM, Trollope AF, et al. A metaanalysis of the prevalence of Low Anterior Resection Syndrome and systematic review of risk factors. Int J Surg 2018;56:234-41.

14. Koda K, Yamazaki M, Shuto K, et al. Etiology and management of low anterior resection syndrome based on the normal defecation mechanism. Surg Today 2019;49:803-8.

15. Pieniowski EHA, Palmer GJ, Juul T, et al. Low Anterior Resection Syndrome and Quality of Life After SphincterSparing Rectal Cancer Surgery: A Long-term Longitudinal Follow-up. Dis Colon Rectum 2019;62:14-20.

16. Temple LKF. Expert Commentary on Low Anterior Resection Syndrome. Dis Colon Rectum 2019;62:1423-4.

17. Juul T, Ahlberg M, Biondo S, et al. International validation of the low anterior resection syndrome score. Ann Surg 2014;259:728-34.

18. Juul T, Elfeki H, Christensen P, et al. Normative Data for the Low Anterior Resection Syndrome Score (LARS Score). Ann Surg 2019;269:1124-8.

19. Kim CW, Jeong WK, Son GM, et al. Validation of Korean Version of Low Anterior Resection Syndrome Score Questionnaire. Ann Coloproctol 2020;36:83-7.

(English Language Editor: B. Draper) 